\title{
Research on the Communication Mode and Marketing Strategy of Mobile Media

\author{
Di Kang
}

Department of Information Management, Hainan College of Software Technology, Qionghai, China jxs1999@163.com

Keywords: Mobile media; Communication mode; Single point; Marketing strategy

\begin{abstract}
Smart phones are the important communication tools and information information dissemination media, and the communication mode of mobile media and the transmission form of mobile phone network affect the development of the mobile phone. Based on the theory of communication and marketing, this paper gives the transmission mode and marketing strategy of mobile media, which is of practical significance for promoting the sound development of mobile media industry.
\end{abstract}

\section{Introduction}

Mobile phone media marketing strategy is based on the research for the theme of the novel and has strong practical significance, and the systematic research and reference theories are also constantly and deeply in recent years and exhibition[1]. Combined with the needs of mobile media audiences, this paper studies the advantages and characteristics of mobile media in the era of media and proposes a constructive marketing strategy of mobile media from the perspective of communication and marketing, which provides a basis for the development of mobile media theoretically, and promotes the healthy development of mobile media industry in the practical application based on the analysis of the transmission characteristics of mobile media.

\section{Mobile Media Communication Mode}

Mobile Media Communication Network. In the dissemination process Mobile media communication not only broke the institutions and authority of the traditional mass media of the subject, but also had the characteristics of strong interpersonal communication[2]. Thus it presented multiple interactive transmission of the main body and the decomposition of concentration of rights in the new communication mode, which broke through the media communication mode. Mobile media communication network is shown in Figure 1.

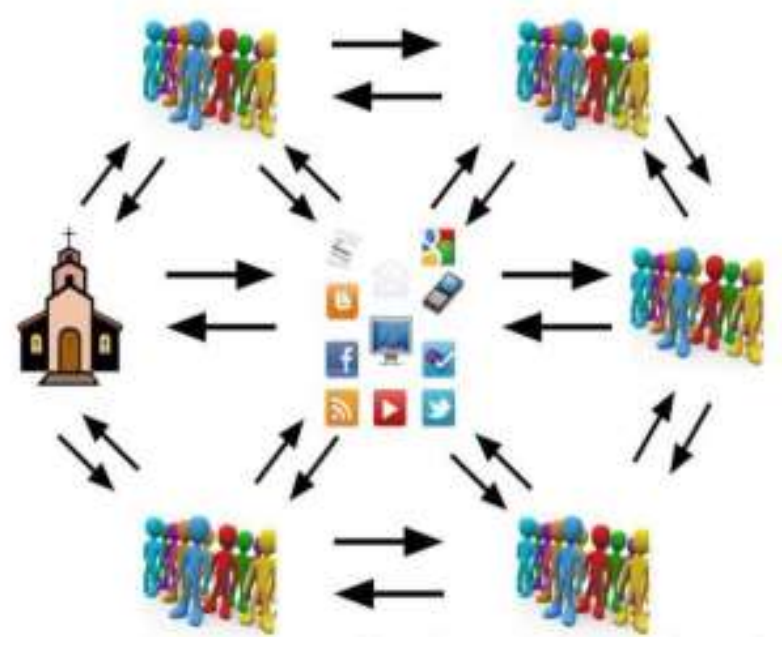

Figure 1. Mobile media communication network 
The Single Point Interpersonal Communication Model. In the single point interpersonal communication model, the individual has the dual identity of the sender of information and the reducers of the information symbol. Mobile phone users can not only obtain content and information through mobile phones, but also establish interpersonal communication channels between user groups. The power of the gateway decomposes the majority of the main body of communication, which is not only multiple and contradictory the subject but also the main body of dispersion.

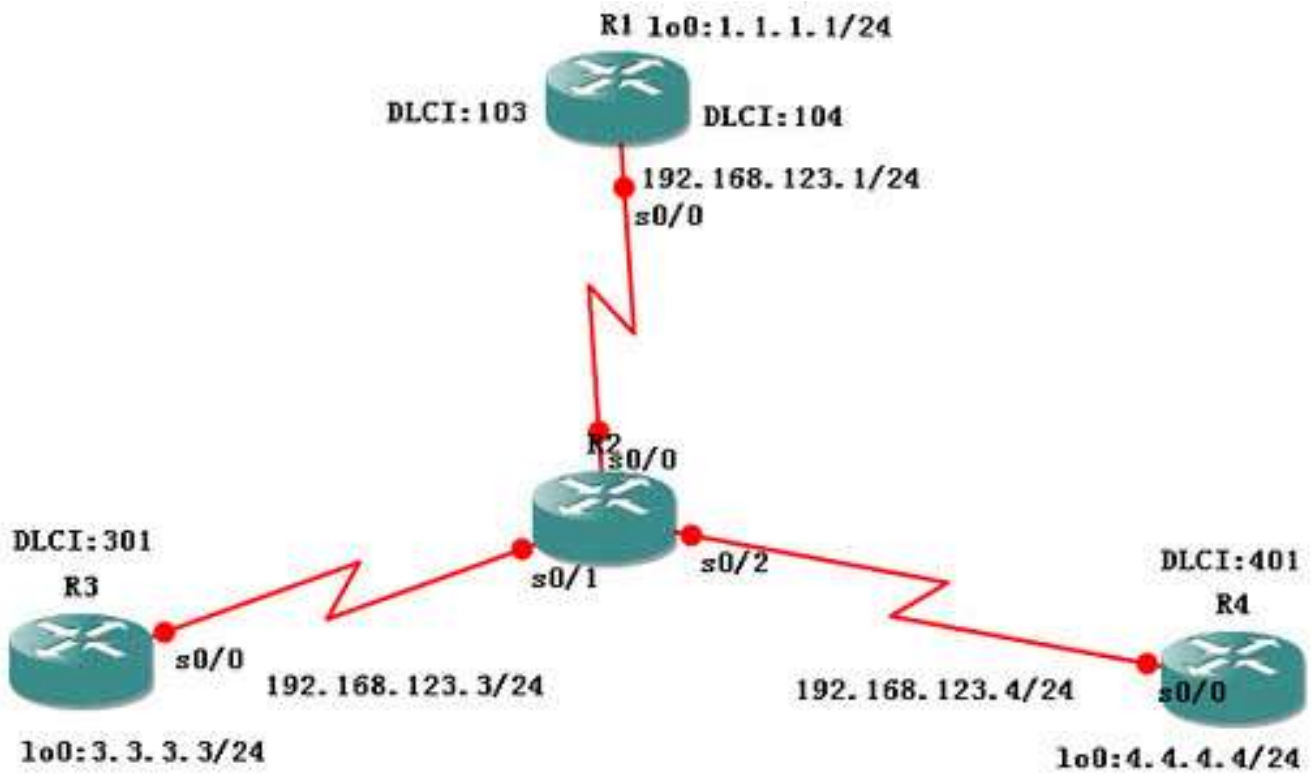

Figure 2. The single point interpersonal communication model

Communication between individuals is interactive, and no other people are involved in information recipients or sender. The process of Information sending, receiving and feedback is fast, and both sides are equally in status. The spread of this single point network has become one of the communication modes of mobile media.

The diffusion model of mass communication. In the diffusion model of mass communication, mobile media will transform a variety of single communication modes of new media communication platform into a complex and comprehensive communication mode. The web site becomes a disseminator that collect and process information, which plays the role of the gateway at the same time. The diffusion model of mass communication is shown in Figure 3.

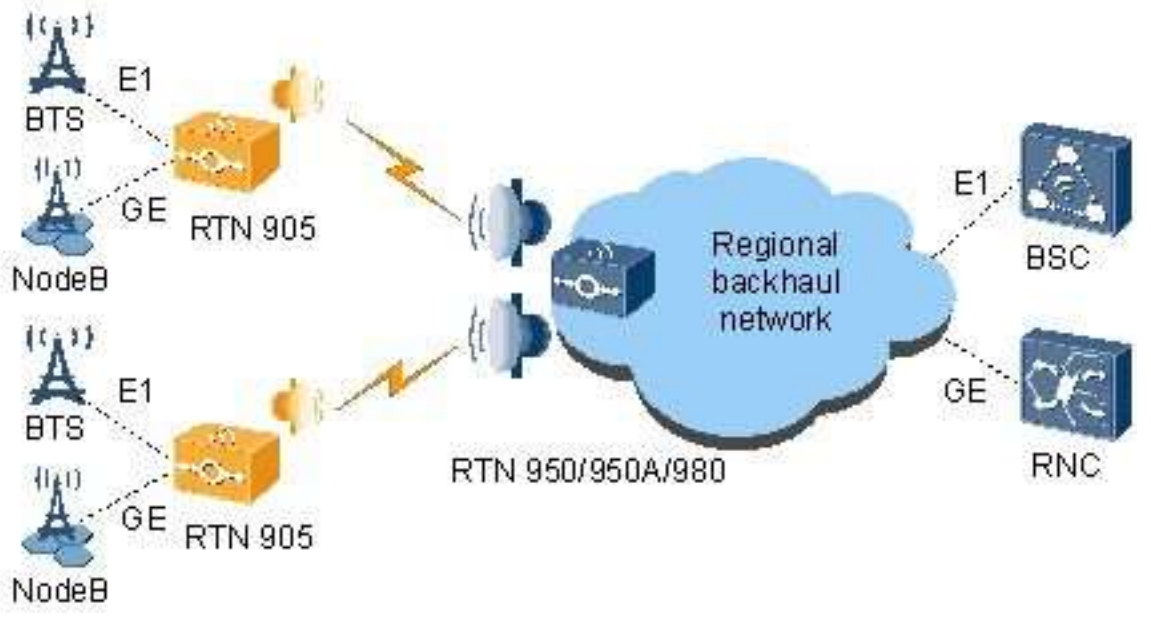

Figure 3. The diffusion model of mass communication

The Comparison of Signal Frame of CP-OFDM and TDS-OFDM. The purpose of this section is to briefly describe the principle of TDS-OFDM modulation to explain its advantages. To combat 
inter-symbol interference (ISI) in the received signal, OFDM adopted the guard interval (GI) principle. By attaching a periodic GI between the transmission symbols, the linear convolution between the input signals and the channel can be transformed into a circular convolution.

$$
s(k)=y(k) / h(k), \quad k=0, \ldots, N_{c}-1
$$

where $y(k)$ is the received signal, $N_{c}$ is the number of subcarriers. The comparison of signal frame of CP-OFDM and TDS-OFDM is shown in Figure 4.

\begin{tabular}{|c|c|c|c|}
\hline $\mathrm{CP}$ & Data \& TPS + Pilots & $\mathrm{CP}$ & Data \& TPS + Pilots \\
\hline \multicolumn{4}{|c|}{$\longleftarrow$ TDS-OFDM Signal Frame $\longrightarrow$} \\
\hline PN & Data \& TPS & $\mathrm{PN}$ & Data \& TPS \\
\hline
\end{tabular}

Figure 4. The comparison of signal frame of CP-OFDM and TDS-OFDM.

Although the CSI can be quickly estimated utilizing the good auto-correlation property of the PN sequence:

$$
\sum_{n=0}^{N_{p}-1} c(n+k) c^{*}(n)=\delta(k)
$$

where $[\cdot]^{*}$ denotes the complex conjugate.

The target channel impulse response (CIR) is

$$
h_{t}(n)=\sum_{p=0}^{N_{p}-1} a_{t p} \delta(n-p)
$$

where $a_{t p}$ is the complex amplitude of the pth delay, and $p$ is the delay normalized to the sampling $\operatorname{period} T, N_{p}$ is the number of channel taps in $\mathbf{h}_{t}$ with $N_{t}$ non-zero components corresponding to $N_{t}$ target delays.

The clutter CIR is

$$
h_{c}(n)=\sum_{l=0}^{N_{p}-1} a_{c l} \delta(n-l)
$$

After down-converted and sampled with period $T$, the received DTTB signals in PBR from ith symbols are written as:

$$
\begin{aligned}
y_{i}(n) & =\sum_{p=0}^{N_{p}-1} h_{t}(p) s_{i}(n-p) e^{j 2 \pi(i-1)\left(\varepsilon_{0}+\varepsilon_{p}\right) n} \\
& +\sum_{l=0}^{N_{p}-1} h_{c}(l) s_{i}(n-l) e^{j 2 \pi(i-1) \varepsilon_{0} n}+w_{i}(n),
\end{aligned}
$$

The angle-of-arrival (AoA) information of target is often unreliable in PBR, where passive location has to be accomplished by an ellipse localization method which requires at least three illuminators. Therefore, a passive location model in a SFN with three illuminators is established, as shown in Figure 5. 


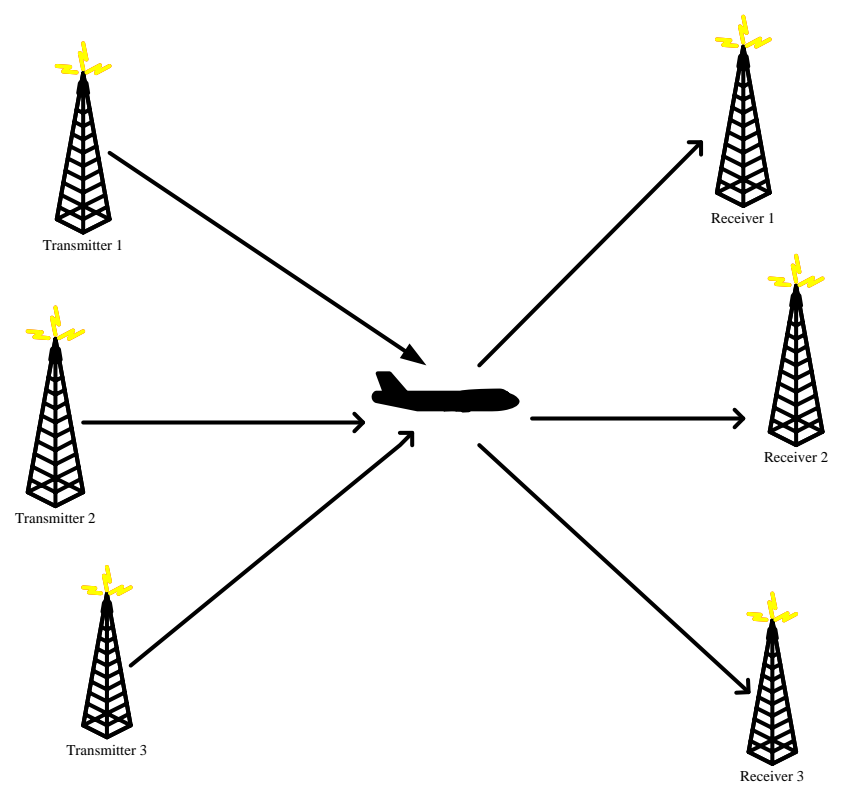

Figure 5. Passive coherent location in SFN

\section{The Partner Selection model of Mobile marketing based on Trust-Degree}

Factors of Ability/Competence. This constituent concern the perceived competent of candidate partner within some specific domain, or its ability to successfully cooperate. They are essential precondition for cooperation in VEs. Partners' skills enable trusting decision. Previous research on firms' reliability has commonly focused on their core competency. Inkpen and Curral summarize five conditions of firm's reliability: prior cooperation, routines, individual inclination, mutual collaboration and evaluation of partners' ability.

Factors of Incentive. This constituent concerns the intention of candidate partner, which is a belief relative to the willingness that they have decided and intended to do or not to do as its cooperators. It allows one to dynamically form an opinion about another entity. While arriving at a trusting decision, one is taking advantage of the trustee's independent goals and, predicting his behavior on such a basis.

Factors of Persistence. The third constituent concerns the perceptibility of behaviors for candidate partners. This is a belief that the cooperator is stable enough in its intentions and both have no serious conflicts within the collaboration. For the candidate partner, it shouldn't been unpredictable by characters. This can be found in interpersonal trust as sincerity and benevolence . By monitoring partners' behaviors, one may form more accurate perceptions of others' trustworthiness.

\section{Summary}

Mobile phone media practitioners should use the advantages of mobile phone media and play characteristics of operation mechanism of mobile phone media. In the tradition of past experience, it brings opportunities and innovation for mobile phone media combined with the characteristics. The mobile phone media operations should be on track and their mobile phone media marketing mode should be built.

\section{References}

[1] Balamurugan, R., Ramakrishnan, V., and Nidur, S., Performance evaluation of a two stage adaptive genetic algorithm in structural topology optimization, Applied Soft Computing. 8 (2008) 1607-1624.

[2] Breite, R., and Vanharanta, H., Evaluating collaboration and core competence in a virtual enterprise, Psych Nology Journal, 1 (2003), 391-399. 